\title{
Parietal hemorrhagic infarction presenting with gastrointestinal symptoms: A case report
}

\author{
Jamir Pitton Rissardo ${ }^{1}$, Ana Letícia Fornari Caprara ${ }^{1}$ \\ (1) Universidade Federal de Santa Maria, Centro de Ciências da Saúde, Santa Maria, Brasil
}

\section{Date submitted:}

Jun 28, 2018

Date accepted:

Sep 28, 2018

Online publication date:

June 15, 2019

\section{Corresponding Author:}

Jamir Pitton Rissardo

Universidade Federal de Santa

Maria, Centro de Ciências da

Saúde, Santa Maria, Brasil,

jamirrissardo@gmail.com

Keywords: Parietal lobe, Stroke,

Abdominal Pain.

\begin{abstract}
The clinical presentation of spontaneous intracerebral hemorrhages $(\mathrm{ICH})$ varies depending upon the location of the hemorrhage. We report a case of an elderly female that was admitted due to diffuse crampy abdominal pain, nausea, and vomiting with one day of onset. Her medical history included hypertension and no previous abdominal or pelvic surgery. Her abdomen was soft, nondistended, and mildly tender to palpation in all quadrants. Her vital signs were normal. Laboratory blood tests and urinalysis were within normal limits. An abdominal CT was unremarkable. On day two of hospital admission, she persisted with the symptoms and started presenting an acute confusional state. Her neurological examination showed hippus. A cranial CT scan showed left parietal hemorrhage. A loading dose of phenytoin was started and her clinical symptoms fully recovered. On the following days, she had good acceptance of oral diet and was discharged to home with phenytoin prophylactically.
\end{abstract}

\section{Introduction}

Abdominal pain corresponds to up to $10 \%$ of all presentations to the emergency department. The range of differential diagnosis is broad and some of the most challenging patients to evaluate are elderly individuals (1). In this context, the clinical presentation of spontaneous intracerebral hemorrhages $(\mathrm{ICH})$ varies and may be atypical depending upon the location of the haemorrhage (2). However, to the author's knowledge, ICH in the parietal lobe presenting with crampy abdominal pain and vomiting has not been reported yet. Hereinafter, we report a case of an elderly woman who had abdominal pain and vomiting probably secondary to parietal hemorrhagic infarction.

\section{Case Report}

A 69-year-old female was admitted to our emergency department due to diffuse crampy abdominal pain, nausea, and vomiting with one day of onset. Her medical history included hypertension and no prior abdominal or pelvic surgery. On physical exam, her abdomen was soft, nondistended, and mildly tender to palpation in all quadrants. Her vital signs (blood pressure, heart rate, respiratory rate, temperature) were normal and sta- ble. Laboratory blood tests and urinalysis were within normal limits. A computed tomography of the abdomen was unremarkable.

On day two of hospital admission, she persisted with the clinical manifestations and started to present an acute confusional state. Her vital signs were stable and arterial blood gas, lactate, and electrolytes were within normal limits. Her neurological examination showed a pupillary hippus. A cranial CT scan showed left parietal hemorrhage and no signs of intracranial hypertension (Figure 1). A loading dose of phenytoin $15 \mathrm{mg} / \mathrm{Kg}$ was started. Full recovery of clinical symptoms was achieved. On the following days, she had good acceptance of oral diet and was discharged to home with phenytoin 100mg 3 times daily.

\section{Discussion}

The examination of the abdomen is subjective. Particularly in elderly individuals, who often tolerate pain at greater degrees and seek medical care with delay, the abdominal exam may not be helpful in identifying life-threatening conditions and early signs of adverse outcomes in abdominal pain. Therefore, careful examination of the other systems may offer valuable addi- 


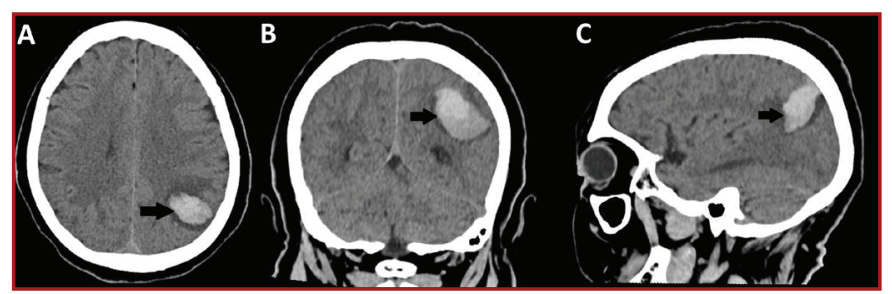

Figure 1. Images of computed tomography scan showing left parietal haemorrhage (indicated by the arrow). Axial (A), coronal (B), and sagittal (C) views of cranial CT scan.

tional clues to the diagnosis (1).

Age and hypertension are the main risk factor for spontaneous $\mathrm{ICH}$. About one-half of the subjects present with headache and vomiting (2). Nevertheless, to the author's knowledge, spontaneous $\mathrm{ICH}$ presenting with cramping abdominal pain and vomiting was not reported until the present moment.

A prospective study that analyzed 63 subjects with $\mathrm{ICH}$ who underwent continuous electroencephalogram monitoring found that, within three days of the hemorrhage, $30 \%$ of the patients presented seizures, which were nonconvulsive in most of the cases (3).

In this way, we hypothesize that seizure activity is involved in the atypical clinical presentation of the parietal hemorrhage seen in this case. In previous studies, it has been suggested that brain hemorrhage may predispose to the occurrence of a pro-epileptogenic foci, which could lead to seizure activity. Therefore, the clinical manifestations found in our patient, such as symptoms of crampy abdominal pain, nausea, vomiting, and the hippus finding could correspond to a seizure. Those symptoms have already been reported with seizure activity in the parietal lobe. In addition, the full recovery after the loading dose of phenytoin supports this hypothesis (4-6). However, one limitation of the reported case is the fact that the subject was not continuously monitored with an electroencephalogram.

In this case, the diagnosis was challenging due to the initial manifestation of acute abdominal pain with the absence of headache. A throughout review of systems and physical examination provided an essential clue to the diagnosis since the identification of the continuous oscillation of the pupillary diameter in the absence of light flux variations or other external stimuli (also known as hippus) prompted the cranial CT scan. Hippus is often a normal finding, but it could occur in pathologic conditions (4).

\section{Acknowledgements}

JPR: study design, data collection, drafting and revising the manuscript. ALFC: study design, drafting and revising the manuscript.

\section{Conflict of Interest}

None of the authors has any conflict of interest to disclose. We confirm that we have read the Journal's position on issues involved in ethical publication and affirm that this report is consistent with those guidelines.

\section{References}

1. Kamin RA, Nowicki TA, Courtney DS, Powers RD. Pearls and pitfalls in the emergency department evaluation of abdominal pain. Emerg Med Clin North Am. 2003;21(1):61-72.
2. Sturgeon JD, Folsom AR, Longstreth W, Shahar E, Rosamond WD, Cushman M. Risk factors for intracerebral hemorrhage in a pooled prospective study. Stroke. 2007;38(10):2718-2725.

3. Vespa PM, O'phelan K, Shah M, et al. Acute seizures after intracerebral hemorrhage $A$ factor in progressive midline shift and outcome. Neurology. 2003;60(9):14411446.

4. Centeno M, Feldmann M, Harrison NA, et al. Epilepsy causing pupillary hippus: an unusual semiology. Epilepsia. 2011;52(8): e93-e96.

5. Moore MT. Paroxysmal abdominal pain: a form of focal symptomatic epilepsy. J Am Med Assoc. 1944;124(9):561-563.

6. Sekimoto M, Kato M, Kaneko Y, Onuma T. Ictal nausea with vomiting as the major symptom of simple partial seizures: Electroencephalographic and magnetoencephalographic analysis. Epilepsy Behav. 2007;11(4):582-587. 